\title{
JACOBI FORMS THAT CHARACTERIZE PARAMODULAR FORMS
}

\author{
TOMOYOSHI IBUKIYAMA, CRIS POOR, AND DAVID S. YUEN
}

\begin{abstract}
The Fourier Jacobi expansions of paramodular forms are characterized from among all formal series of Jacobi forms by two conditions on the Fourier coefficients of the Jacobi forms: a growth condition and a set of linear relations. Examples, both theoretical and computational, indicate that the growth condition may be superfluous.
\end{abstract}

\section{INTRODUCTION}

For theoretical purposes it would be nice to characterize the Fourier Jacobi expansions of Siegel paramodular forms of degree two from among all formal power series with Jacobi forms as coefficients. For computational purposes it would be nice if the characterization were in terms of linear relations among the Fourier coefficients of the various Jacobi forms. We achieve this goal only in a few cases.

The linear relations we study arise from a symmetry possessed by the Fourier Jacobi expansions of paramodular forms. Let $J_{k, m}$ denote the complex vector space of Jacobi forms of weight $k$ and index $m$. Let $\Gamma$ be a group commensurable with $\mathrm{Sp}_{2}(\mathbb{Z})$ and denote by $M_{k}(\Gamma)$ the complex vector space of Siegel modular forms of weight $k$ automorphic with respect to $\Gamma$. One commensurable family is given by the paramodular groups $K(N)$ :

$$
K(N)=\left(\begin{array}{cccc}
* & N * & * & * \\
* & * & * & * / N \\
* & N * & * & * \\
N * & N * & N * & *
\end{array}\right) \cap \operatorname{Sp}_{2}(\mathbb{Q}), \text { where } * \in \mathbb{Z} .
$$

Each paramodular form $f \in M_{k}(K(N))$ has a Fourier Jacobi expansion $f\left(\begin{array}{cc}\tau & z \\ z & \omega\end{array}\right)=\sum_{m \geq 0: N \mid m} \phi_{m}(\tau, z) e(m \omega)$ where $\left(\begin{array}{cc}\tau & z \\ z & \omega\end{array}\right)$ is in the Siegel upper half space and $\phi_{m} \in J_{k, m}$. These Jacobi forms $\phi_{m}$ are not independent and possess a symmetry that is best expressed by using a normalizer $\mu_{N}$

Date: November 30, 2018.

2000 Mathematics Subject Classification. Primary 11F46, 11F50.

Key words and phrases. Jacobi forms, Paramodular forms. 
of the paramodular group $K(N)$ satisfying $\mu_{N}^{2}=-I_{4}$ and given by $\mu_{N}=\left(\begin{array}{cc}-F_{N}^{\prime} & 0 \\ 0 & F_{N}\end{array}\right)$, where the Fricke involution $F_{N}=\frac{1}{\sqrt{N}}\left(\begin{array}{cc}0 & 1 \\ -N & 0\end{array}\right)$ is the usual normalizer of $\Gamma_{0}(N)=\left\{\left(\begin{array}{ll}a & b \\ c & d\end{array}\right) \in \mathrm{SL}_{2}(\mathbb{Z}): N \mid c\right\}$.

For $\epsilon= \pm 1$, let $M_{k}(K(N))^{\epsilon}=\left\{f \in M_{k}(K(N)):\left.f\right|_{k} \mu_{N}=\epsilon f\right\}$ be the plus and minus eigenspaces of $\mu_{N}$. Let the Fourier Jacobi expansion map, FJ : $M_{k}(K(N))^{\epsilon} \rightarrow \prod_{m \in \mathbb{Z}: m \geq 0, N \mid m} J_{k, m}$, be defined by $\mathrm{FJ}(f)=$ $\sum_{m: N \mid m} \phi_{m} \xi^{m}$ and write, for $(\tau, z) \in \mathcal{H}_{1} \times \mathbb{C}$,

$$
\phi_{m}(\tau, z)=\sum_{n, r \in \mathbb{Z}: 4 m n \geq r^{2}, n \geq 0} c\left(n, r ; \phi_{m}\right) e(n \tau+r z) .
$$

These coefficients possess the symmetry

$$
c\left(n, r ; \phi_{m}\right)=\epsilon c\left(m / N,-r ; \phi_{n N}\right) .
$$

We mention that $f \in M_{k}(K(N))^{\epsilon}$ is a cusp form if and only if $\mathrm{FJ}(f) \in \prod_{m \in \mathbb{Z} ; m \geq 0, N \mid m} J_{k, m}^{\text {cusp }}$. This nontrivial assertion follows from the representation of the one-dimensional cusps by matrices of the shape $\left(\begin{array}{ll}A & 0 \\ 0 & D\end{array}\right)$. In fact, the one-dimensional cusps correspond to divisors $t$ of $N$ via $D^{*}=A=\left(\begin{array}{ll}1 & t \\ 0 & 1\end{array}\right)$, see Reefschläger [18] or compare [17].

In Theorem 2.2 we show that certain convergent series of Jacobi forms satisfying the symmetry (11) are in fact the Fourier Jacobi expansion of some Siegel paramodular form. However, the real question motivating this article is: Are formal series of Jacobi forms satisfying the symmetry (11) the Fourier Jacobi expansions of Siegel paramodular forms? Work of H. Aoki [1] essentially answers this question affirmatively for $N=1$ and we prove this for $N \in\{2,3,4\}$ as well by following his method. Let us give a more definite formulation.

Definition 1.1. Let $\mathcal{X}_{2}^{\text {semi }}(N)=\left\{\left(\begin{array}{ll}a & b \\ b & c\end{array}\right) \geq 0: a, 2 b, c \in \mathbb{Z}\right.$ and $\left.N \mid c\right\}$ for $N \in \mathbb{N}$. For $k \in \mathbb{Z}$, let $\Phi=\sum_{m: N \mid m} \phi_{m} \xi^{m} \in \prod_{m \geq 0: N \mid m} J_{k, m}$ be a formal power series whose coefficients are Jacobi forms. For $\epsilon \in\{-1,1\}$, we say that $\Phi$ satisfies the Involution $(\epsilon)$ condition if

$$
\forall\left(\begin{array}{cc}
n & r / 2 \\
r / 2 & m
\end{array}\right) \in \mathcal{X}_{2}^{\text {semi }}(N), c\left(n, r ; \phi_{m}\right)=\epsilon c\left(\frac{m}{N},-r ; \phi_{n N}\right) .
$$

We say that $\Phi$ satisfies the growth condition if

$$
\forall \rho>1, \exists A>0: \forall\left(\begin{array}{cc}
n & r / 2 \\
r / 2 & m
\end{array}\right) \in \mathcal{X}_{2}^{\text {semi }}(N),\left|c\left(n, r ; \phi_{m}\right)\right| \leq A \rho^{n+m} .
$$

Set $\mathbb{M}_{k}(N)^{\epsilon}=\left\{\Phi \in \prod_{m \geq 0: N \mid m} J_{k, m}: \Phi\right.$ satisfies Involution $\left.(\epsilon)\right\}$.

We would like to know when the map FJ : $M_{k}(K(N))^{\epsilon} \rightarrow \mathbb{M}_{k}(N)^{\epsilon}$ is surjective. In Theorem 2.2 we show that this map surjects onto the subspace of $\mathbb{M}_{k}(N)^{\epsilon}$ that satisfies the growth condition, thereby giving at least one theoretical characterization of the Fourier Jacobi 
expansions of Siegel paramodular forms. Details aside, this amounts to the fact that the paramodular groups are generated by the Jacobi group and an involution. By following Aoki's method however, we do prove the surjectivity of FJ onto $\mathbb{M}_{k}(N)^{\epsilon}$ for $N \leq 4$.

Theorem 1.2. Let $N \in\{1,2,3,4\}$ and $\epsilon \in\{-1,1\}$. For all weights $k \in \mathbb{Z}$, the Fourier Jacobi expansion map FJ from paramodular forms to formal series of Jacobi forms that satisfy the Involution $(\epsilon)$ condition, FJ : $M_{k}(K(N))^{\epsilon} \rightarrow \mathbb{M}_{k}(N)^{\epsilon}$, is an isomorphism.

As a corollary we obtain new results for the generating functions of the plus and minus eigenspaces. For any prime $p, \operatorname{dim} S_{k}(K(p))$ is known in [10] for $k>4$, in [12] for $k=3$, 4, and for $p<349$ and $k=2$ in [16]. We can easily show that the generalized Siegel $\Phi$ operator, the projection from $M_{k}(K(N))$ to the boundary of the Satake compactification, is always surjective for any $k$ for squarefree $N$. Indeed, this is due to Satake [19] when $k>4$, and, again for squarefree $N$, the image is zero dimensional for $k=2$ and at most one dimensional for $k=4$ due to the known cusp configuration in [11] for prime level and in [17] for general $N$; furthermore, the lift of the Jacobi Eisenstein series of $J_{4, N}$ surjects to the image of $\Phi$ when $k=4$. So the generating function for $\operatorname{dim} M_{k}(K(p))$ can be easily given for any $p$ as long as we know $\operatorname{dim} S_{2}(K(p))$. In fact, the full generating functions are known for $N=2$ by T. Ibukiyama and F. Onodera [13], the plus and minus eigenspaces being given there also, and for $N=3$ by T. Dern [4]. Our proofs use their results. The generating function $\sum \operatorname{dim} M_{k}(K(4)) t^{k}$ is given here for the first time by relying on the definitive results of Igusa [14] for subgroups of $\Gamma_{2}$ that contain the principal subgroup $\Gamma_{2}(2)$. These new results are: 


$$
\begin{aligned}
& \sum_{k \in \mathbb{Z}} \operatorname{dim} M_{k}(K(2))^{+} t^{k}=\frac{1+t^{10}+t^{23}+t^{33}}{\left(1-t^{4}\right)\left(1-t^{6}\right)\left(1-t^{8}\right)\left(1-t^{12}\right)}, \\
& \sum_{k \in \mathbb{Z}} \operatorname{dim} M_{k}(K(2))^{-} t^{k}=\frac{t^{11}+t^{12}+t^{21}+t^{22}}{\left(1-t^{4}\right)\left(1-t^{6}\right)\left(1-t^{8}\right)\left(1-t^{12}\right)}, \\
& \sum_{k \in \mathbb{Z}} \operatorname{dim} M_{k}(K(3))^{+} t^{k}=\frac{1+t^{8}+t^{10}+t^{21}+t^{23}+t^{31}}{\left(1-t^{4}\right)\left(1-t^{6}\right)^{2}\left(1-t^{12}\right)}, \\
& \sum_{k \in \mathbb{Z}} \operatorname{dim} M_{k}(K(3))^{-} t^{k}=\frac{t^{9}+t^{11}+t^{12}+t^{19}+t^{20}+t^{22}}{\left(1-t^{4}\right)\left(1-t^{6}\right)^{2}\left(1-t^{12}\right)} \\
& \sum_{k \in \mathbb{Z}} \operatorname{dim} M_{k}(K(4))^{+} t^{k}=\frac{1+t^{6}+t^{8}+t^{10}+t^{19}+t^{21}+t^{23}+t^{29}}{\left(1-t^{4}\right)^{2}\left(1-t^{6}\right)\left(1-t^{12}\right)} \\
& \sum_{k \in \mathbb{Z}} \operatorname{dim} M_{k}(K(4))^{-} t^{k}=\frac{t^{7}+t^{9}+t^{11}+t^{12}+t^{17}+t^{18}+t^{20}+t^{22}}{\left(1-t^{4}\right)^{2}\left(1-t^{6}\right)\left(1-t^{12}\right)} .
\end{aligned}
$$

The question of the surjectivity of FJ : $M_{k}(K(N))^{\epsilon} \rightarrow \mathbb{M}_{k}(N)^{\epsilon}$ is not idle and has applications to the computation of paramodular forms. To illustrate this, in section 4 we use the symmetry condition to compute $S_{4}(K(31))^{ \pm}$. These computations at least make it plausible that the growth condition is superfluous. Here one may also find a lemma showing that, for prime $p$, initial Fourier Jacobi expansions $\pi_{p J} \circ \mathrm{FJ}: S_{k}(K(p))^{\epsilon} \rightarrow \prod_{j=1}^{J} J_{k, p j}^{\text {cusp }}$ inject for $J \geq\left\llcorner\frac{k}{10}\left(\frac{p^{2}+1}{p+1}\right)\right\lrcorner$.

We thank Nils Skoruppa for his explanations to us about theta blocks. We thank Armand Brumer for suggesting that Fourier Jacobi expansions be used to compute spaces of paramodular cusp forms.

\section{A Characterization of Fourier Jacobi Expansions}

For a ring $R$, let $\operatorname{Sp}_{n}(R)=\left\{\sigma \in \mathrm{GL}_{2 n}(R): \sigma^{\prime} J \sigma=J\right\}$ define the symplectic group over $R$, where $J=\left(\begin{array}{cc}0 & I_{n} \\ -I_{n} & 0\end{array}\right)$ and $\sigma^{\prime}$ is the transpose of $\sigma$. The paramodular group $K(N)$, defined in the Introduction, is generated by the translations $\left(\begin{array}{ll}I & S \\ 0 & I\end{array}\right)$ with $S=\left(\begin{array}{cc}\alpha & \beta \\ \beta & \gamma / N\end{array}\right)$ for $\alpha, \beta, \gamma \in \mathbb{Z}$, and the element $J(N)$, see 3], Theorem 9,

$$
J(N)=\left(\begin{array}{cccc}
0 & 0 & 1 & 0 \\
0 & 0 & 0 & 1 / N \\
-1 & 0 & 0 & 0 \\
0 & -N & 0 & 0
\end{array}\right)
$$


Let $\mathcal{H}_{n}$ denote the Siegel upper half space. For $k \in \mathbb{Z}$, the paramodular forms of weight $k$, denoted by $M_{k}(K(N))$, are the $\mathbb{C}$-vector space of holomorphic $f: \mathcal{H}_{2} \rightarrow \mathbb{C}$ with the property that $\left.f\right|_{k} \sigma=f$ for all $\sigma \in K(N)$. The subspace of cusp forms is given by $S_{k}(K(N))=$ $\left\{f \in M_{k}(K(N)): \forall \sigma \in \operatorname{Sp}_{2}(\mathbb{Z}), \Phi\left(\left.f\right|_{k} \sigma\right)=0\right\}$. Here the slash action, $\left(\left.f\right|_{k}\left(\begin{array}{ll}A & B \\ C & D\end{array}\right)\right)(\Omega)=\operatorname{det}(C \Omega+D)^{-k} f\left((A \Omega+B)(C \Omega+D)^{-1}\right)$ and the $\Phi$ operator, $(\Phi f)(\tau)=\lim _{\lambda \rightarrow+\infty} f\left(\begin{array}{cc}i \lambda & 0 \\ 0 & \tau\end{array}\right)$, are the usual ones, see [6]. Since $\mu_{N}^{2}$ acts trivially on modular forms, we may decompose paramodular forms into plus and minus forms: $M_{k}(K(N))=M_{k}(K(N))^{+} \oplus$ $M_{k}(K(N))^{-}$where $M_{k}(K(N))^{\epsilon}=\left\{f \in M_{k}(K(N)): f \mid \mu=\epsilon f\right\}$ for $\epsilon \in\{-1,1\}$.

Every paramodular form $f \in M_{k}(K(N))$ has a Fourier expansion

$$
f(\Omega)=\sum_{T \in \mathcal{X}_{2}^{\text {semi }}(N)} a(T ; f) e(\langle\Omega, T\rangle)
$$

supported on $\mathcal{X}_{2}^{\text {semi }}(N)=\left\{\left(\begin{array}{ll}a & b \\ b & c\end{array}\right) \geq 0: a, 2 b, c \in \mathbb{Z}\right.$ and $\left.N \mid c\right\}$; here $e(z)=e^{2 \pi i z}$ and $\langle A, B\rangle=\operatorname{tr}(A B)$. Setting $T[\sigma]=\sigma^{\prime} T \sigma$, we additionally have $a(T[\sigma] ; f)=\operatorname{det}(\sigma)^{k} a(T ; f)$ for all $\sigma \in \hat{\Gamma}^{0}(N)=\left\{\left(\begin{array}{ll}a & b \\ c & d\end{array}\right) \in\right.$ $\left.\mathrm{GL}_{2}(\mathbb{Z}): N \mid b\right\}$. Note that the action of $\hat{\Gamma}^{0}(N)$ stabilizes $\mathcal{X}_{2}^{\text {semi }}(N)$. If we write $\Omega=\left(\begin{array}{cc}\tau & z \\ z & \omega\end{array}\right) \in \mathcal{H}_{2}$ and collect the Fourier expansion of $f$ in powers of $\xi=e(\omega)$, then we obtain the Fourier Jacobi expansion of $f$ : $f\left(\begin{array}{cc}\tau & z \\ z & \omega\end{array}\right)=\sum_{m \geq 0: N \mid m} \phi_{m}(\tau, z) \xi^{m}$ where the

$$
\phi_{m}(\tau, z)=\sum_{n, r \in \mathbb{Z}:\left(\begin{array}{cc}
n & r / 2 \\
r / 2 & m
\end{array}\right) \geq 0, n \geq 0} a\left(\left(\begin{array}{cc}
n & r / 2 \\
r / 2 & m
\end{array}\right) ; f\right) e(n \tau) e(r z)
$$

are Jacobi forms of weight $k$ and index $m$. This Fourier Jacobi expansion is term by term invariant under the group,

$$
\Gamma_{\infty}(\mathbb{Z})=\left(\begin{array}{llll}
* & 0 & * & * \\
* & * & * & * \\
* & 0 & * & * \\
0 & 0 & 0 & *
\end{array}\right) \cap \operatorname{Sp}_{2}(\mathbb{Z}),
$$

and this is one motivation for the definition of Jacobi forms.

Definition 2.1. Let $k, m \in \mathbb{Z}_{\geq 0}$. The $\mathbb{C}$-vector space $J_{k, m}$ of Jacobi forms of weight $k$ and index $m$ is the set of holomorphic $\phi: \mathcal{H}_{1} \times \mathbb{C} \rightarrow \mathbb{C}$ satisfying:

1.) $\forall \sigma \in \Gamma_{\infty}(\mathbb{Z}),\left.\tilde{\phi}\right|_{k} \sigma=\tilde{\phi}$, where $\tilde{\phi}: \mathcal{H}_{2} \rightarrow \mathbb{C}$ is defined by $\tilde{\phi}\left(\begin{array}{cc}\tau & z \\ z & \omega\end{array}\right)=$ $\phi(\tau, z) e(m \omega)$.

2.) Setting $q=e(\tau)$ and $\zeta=e(z)$, the Fourier series of $\phi$ has the form: $\phi(\tau, z)=\sum_{n, r \in \mathbb{Z}: n \geq 0,4 m n \geq r^{2}} c(n, r ; \phi) q^{n} \zeta^{r}$. 
The vector space of Jacobi cusp forms $J_{k, m}^{\text {cusp }}$ is defined by replacing $4 m n \geq r^{2}$ by $4 m n>r^{2}$ in item 2 . If we identify a sequence $\left(\phi_{m}\right) \in$ $\prod_{m \in \mathbb{Z}: m \geq 0, N \mid m} J_{k, m}$ with the formal power series $\sum_{m: N \mid m} \phi_{m} \xi^{m}$, then developing the Fourier Jacobi expansion of a paramodular form as in (2) defines a map FJ : $M_{k}(K(N)) \rightarrow \prod_{m \geq 0: N \mid m} J_{k, m}$. Now we can state a characterization.

Theorem 2.2. Let $k \in \mathbb{Z}_{\geq 0}, N \in \mathbb{N}$ and $\epsilon \in\{-1,1\}$. Let $\Phi=$ $\sum_{m: N \mid m} \phi_{m} \xi^{m} \in \prod_{m \geq 0: N \mid m} J_{k, m}$ be a formal power series whose coefficients are Jacobi forms. There is an $f \in M_{k}(K(N))^{\epsilon}$ such that $\Phi=\mathrm{FJ}(f)$ if and only if $\Phi$ satisfies the Involution $(\epsilon)$ condition and the growth condition of Definition 1.1.

Proof. We first assume that $\Phi=\mathrm{FJ}(f)$ for $f \in M_{k}(K(N))^{\epsilon}$ and write each $T \in \mathcal{X}_{2}^{\mathrm{semi}}(N)$ as $T=\left(\begin{array}{cc}n & r / 2 \\ r / 2 & m\end{array}\right)$. For any $\rho>1$, take $\lambda>0$ with $\rho=e^{2 \pi \lambda}$. By the Koecher principle there is an $A>0$ such that $|f(\Omega)| \leq A$ on $\left\{\Omega=x+i Y \in \mathcal{H}_{2}: Y>\frac{\lambda}{2} I_{2}\right\}$. For $\Omega=X+i \lambda I_{2}$ we have the growth condition:

$$
\begin{array}{r}
\left|c\left(n, r ; \phi_{m}\right)\right|=|a(T ; f)|=\left|\int_{X \in[0,1]^{3}} f(\Omega) e(-\langle\Omega, T\rangle) d X\right| \\
\leq \int_{X \in[0,1]^{3}}|f(\Omega)| e^{2 \pi\left\langle\lambda I_{2}, T\right\rangle} d X \leq A \rho^{\operatorname{tr}(T)}=A \rho^{m+n} .
\end{array}
$$

For the Involution $(\epsilon)$ condition, we need to know the action of the involution $\mu_{N}$ on the Fourier expansion of $f$ :

$$
\begin{aligned}
\left(f \mid \mu_{N}\right)(\Omega)=\operatorname{det}\left(F_{n}\right)^{-k} & \sum a(T ; f) e\left(\left\langle F_{N}^{\prime} \Omega F_{N}, T\right\rangle\right) \\
= & \sum a\left(F_{N} T F_{N}^{\prime} ; f\right) e(\langle\Omega, T\rangle) .
\end{aligned}
$$

Now $F_{N} T F_{N}^{\prime}=\frac{1}{\sqrt{N}}\left(\begin{array}{cc}0 & 1 \\ -N & 0\end{array}\right)\left(\begin{array}{cc}n & r / 2 \\ r / 2 & m\end{array}\right)\left(\begin{array}{cc}0 & -N \\ 1 & 0\end{array}\right) \frac{1}{\sqrt{N}}=\left(\begin{array}{cc}m / N & -r / 2 \\ -r / 2 & N n\end{array}\right)$, so that we have the Involution $(\epsilon)$ condition:

$$
\begin{aligned}
c\left(n, r ; \phi_{m}\right) & =a\left(\left(\begin{array}{cc}
n & r / 2 \\
r / 2 & m
\end{array}\right) ; f\right)=\epsilon a\left(\left(\begin{array}{cc}
n & r / 2 \\
r / 2 & m
\end{array}\right) ; f \mid \mu_{N}\right) \\
& =\epsilon a\left(\left(\begin{array}{cc}
m / N & -r / 2 \\
-r / 2 & N n
\end{array}\right) ; f\right)=\epsilon c\left(\frac{m}{N},-r ; \phi_{N n}\right) .
\end{aligned}
$$

Now assume that $\Phi=\sum \phi_{m} \xi^{m}$ satisfies the growth and $\operatorname{Involution}(\epsilon)$ conditions. For any $T=\left(\begin{array}{cc}n & r / 2 \\ r / 2 & m\end{array}\right) \in \mathcal{X}_{2}^{\text {semi }}(N)$, define $a(T)$ by $a(T)=$ $c\left(n, r ; \phi_{m}\right)$. On the set $\left\{\Omega=x+i Y \in \mathcal{H}_{2}: Y \geq \lambda I_{2}\right\}$ the series $\sum_{T \in \mathcal{X}_{2}^{\text {semi }}(N)} a(T) e(\langle\Omega, T\rangle)$ is majorized by a convergent series of constants. To see this, choose $\rho$ with $1<\rho<e^{2 \pi \lambda}$ so that by the growth 
condition there is an $A>0$ with $|a(T)|=\left|c\left(n, r ; \phi_{m}\right)\right| \leq A \rho^{n+m}$ and so

$$
\begin{aligned}
\sum|a(T)| e^{-2 \pi\langle Y, T\rangle} & \leq \sum A \rho^{m+n} e^{-2 \pi\langle Y, T\rangle}=A \sum_{T}\left(\frac{\rho}{e^{2 \pi \lambda}}\right)^{m+n} \\
& \leq A \sum_{n=0}^{\infty} \sum_{m=0}^{\infty}(2 n+2 m+1)\left(\frac{\rho}{e^{2 \pi \lambda}}\right)^{m+n}
\end{aligned}
$$

Since the convergence is uniform on compact sets, we may define a holomorphic function $f: \mathcal{H}_{2} \rightarrow \mathbb{C}$ via $f(\Omega)=\sum_{T \in \mathcal{X}_{2}^{\text {semi }}(N)} a(T) e(\langle\Omega, T\rangle)$.

The absolute convergence of this series shows that $f\left(\begin{array}{cc}\tau & z \\ z & \omega\end{array}\right)$ is equal to the rearrangement $\sum_{m \in \mathbb{Z}_{\geq 0}: N \mid m} \phi_{m}(\tau, z) e(m \omega)$, or $f=\sum_{m \in \mathbb{Z}_{\geq 0}: N \mid m} \tilde{\phi}_{m}$. The invariance of $f$ under the action of the group $\Gamma_{\infty}(\mathbb{Z})$ now follows from the invariance of the $\tilde{\phi}_{m}$. In particular, we have $f \mid E_{1}=f$ for

$$
E_{1}=\left(\begin{array}{cccc}
0 & 0 & 1 & 0 \\
0 & 1 & 0 & 0 \\
-1 & 0 & 0 & 0 \\
0 & 0 & 0 & 1
\end{array}\right) \in \Gamma_{\infty}(\mathbb{Z})
$$

Furthermore, the Involution $(\epsilon)$ condition gives us

$$
a\left(F_{N} T F_{N}^{\prime}\right)=c\left(\frac{m}{N},-r ; \phi_{n N}\right)=\epsilon c\left(n, r ; \phi_{m}\right)=\epsilon a(T),
$$

so that

$$
\begin{aligned}
\left(\left.f\right|_{k} \mu_{N}\right)(\Omega) & =\operatorname{det}\left(F_{n}\right)^{-k} \sum_{T \in \mathcal{X}_{2}^{\mathrm{semi}}(N)} a(T) e\left(\left\langle F_{N}^{\prime} \Omega F_{N}, T\right\rangle\right) \\
= & \sum_{T} a\left(F_{N} T F_{N}^{\prime}\right) e(\langle\Omega, T\rangle)=\sum_{T} \epsilon a(T) e(\langle\Omega, T\rangle)=\epsilon f(\Omega) .
\end{aligned}
$$

Following Gritsenko [8], we have $f\left|E_{1} \mu_{N}=f\right| \mu_{N}=\epsilon f$ and therefore that $f \mid\left(E_{1} \mu_{N}\right)^{2}=f$. The group $K(N)$ is generated by translations and the element $\left(E_{1} \mu_{N}\right)^{2}=-J(N)$ so that $f \in M_{k}(K(N))^{\epsilon}$.

\section{AOKI's METHOD FOR $N=2,3$ AND 4 .}

Does Theorem 2.2 remain true without the growth condition? A method of H. Aoki [1] shows that it does for $N=1$. We successfully use Aoki's method to show the same for $N \leq 4$. 
Definition 3.1. Let $j, k, m \in \mathbb{Z}, N \in \mathbb{N}$ and $\epsilon \in\{-1,1\}$. Set

$$
\begin{aligned}
\mathbb{M}_{k}^{(j)}(N)^{\epsilon} & =\left\{\Phi=\sum_{m \in \mathbb{Z}: m \geq N j: N \mid m} \phi_{m} \xi^{m} \in \mathbb{M}_{k}(N)^{\epsilon}\right\}, \\
\operatorname{ord} \phi & =\min \left\{n \in \mathbb{Z}_{\geq 0}: \exists r \in \mathbb{Z}: c(n, r ; \phi) \neq 0\right\}, \text { for } \phi \in J_{k, m}, \\
J_{k, m}(j) & =\left\{\phi \in J_{k, m}: \operatorname{ord} \phi \geq j\right\} .
\end{aligned}
$$

Here, as in Aoki [1] [2], precise dimensions in specific cases follow from inequalities that are in general too generous. Most dramatically, the final terms in the following Estimate diverge for $N>5$ and large weights.

Lemma 3.2. (Estimate) Let $N \in \mathbb{N}, \in \in\{-1,1\}, k \in \mathbb{Z}$ and set $\delta=\lg \left((-1)^{k} \epsilon\right)=\left\{\begin{array}{ll}0, & \text { if }(-1)^{k} \epsilon=1 \\ 1, & \text { if }(-1)^{k} \epsilon=-1 .\end{array}\right.$ We have $\operatorname{dim} M_{k}(K(N))^{\epsilon} \leq \operatorname{dim} \mathbb{M}_{k}(N)^{\epsilon} \leq \sum_{j=0}^{\infty} \operatorname{dim}\left(\mathbb{M}_{k}^{(j)}(N)^{\epsilon} / \mathbb{M}_{k}^{(j+1)}(N)^{\epsilon}\right) \leq$ $\sum_{j=0}^{\infty} \operatorname{dim} J_{k, N j}(j+\delta) \leq \begin{cases}\sum_{j=0}^{\infty} \sum_{i=0}^{N j} \operatorname{dim} M_{k+2 i-12(j+\delta)}, & k \text { even } \\ \sum_{j=1}^{\infty} \sum_{i=1}^{N j-1} \operatorname{dim} M_{k-1+2 i-12(j+\delta)}, & k \text { odd }\end{cases}$

(For $k$ odd, $N=j=1$ gives an empty second sum.)

Proof. The first inequality follows since FJ : $M_{k}(K(N))^{\epsilon} \rightarrow \mathbb{M}_{k}(N)^{\epsilon}$ is injective, the second by the filtration $\mathbb{M}_{k}^{(j)}(N)^{\epsilon} \supseteq \mathbb{M}_{k}^{(j+1)}(N)^{\epsilon}$. For the third, consider the exact sequence

$$
0 \hookrightarrow \mathbb{M}_{k}^{(j+1)}(N)^{\epsilon} \hookrightarrow \mathbb{M}_{k}^{(j)}(N)^{\epsilon} \rightarrow J_{k, N j}
$$

where the final map sends $\Phi=\sum_{i=j}^{\infty} \phi_{i N} q^{i N}$ to $\phi_{j N}$. The $\operatorname{Involution}(\epsilon)$ condition shows that the image of the last map is inside $J_{k, N j}(j+\delta)$. This is the obvious but important point. If $\Phi \in \mathbb{M}_{k}^{(j)}(N)^{\epsilon}$ then for all $\ell<j$ we have $\phi_{N \ell}=0$, so that $c\left(\ell, r ; \phi_{N j}\right)=\epsilon c\left(j,-r ; \phi_{N \ell}\right)=0$ and $\phi_{N j} \in J_{k, N j}(j)$. Furthermore, if $(-1)^{k} \epsilon=-1$ then $c\left(j, r ; \phi_{N j}\right)=$ $\epsilon c\left(j,-r ; \phi_{N j}\right)=(-1)^{k} \epsilon c\left(j, r ; \phi_{N j}\right)=-c\left(j, r ; \phi_{N j}\right)$, so $c\left(j, r ; \phi_{N j}\right)=0$ and $\phi_{N j} \in J_{k, N j}(j+1)$. Thus we may uniformly write $\phi_{N j} \in J_{k, N j}(j+\delta)$.

The last inequality follows from Lemma 3 on page 583 in Aoki [1], a consequence of the theory of differential operators in [5]:

$$
\operatorname{dim} J_{k, m}(j) \leq \begin{cases}\sum_{i=0}^{m} \operatorname{dim} M_{k+2 i-12 j}, & \text { if } k \text { even, } \\ \sum_{i=1}^{m-1} \operatorname{dim} M_{k-1+2 i-12 j}, & \text { if } k \text { odd, } m \geq 2 \\ 0, & \text { if } k \text { odd, } m \leq 1\end{cases}
$$


Lemma 3.3. For $N \in\{1,2,3,4,5\}$ and $\epsilon \in\{-1,1\}$, let:

$$
\begin{aligned}
& E_{N, \delta}=\sum_{k \text { even }}\left(\sum_{j=0}^{\infty} \sum_{i=0}^{N j} \operatorname{dim} M_{k+2 i-12(j+\delta)}\right) t^{k}, \\
& D_{N, \delta}=\sum_{k \text { odd }}\left(\sum_{j=1}^{\infty} \sum_{i=1}^{N j-1} \operatorname{dim} M_{k+2 i-12(j+\delta)}\right) t^{k} .
\end{aligned}
$$

We have $E_{1,0}=\left(\left(1-t^{4}\right)\left(1-t^{6}\right)\left(1-t^{10}\right)\left(1-t^{12}\right)\right)^{-1}, D_{1,1}=E_{1,1}=0$ and $D_{1,0}=t^{35} E_{1,0}$. For $2 \leq N \leq 5$ we have

$$
\begin{aligned}
& E_{N, \delta}=t^{12 \delta} \frac{1+t^{10}+t^{8}+\cdots+t^{14-2 N}}{\left(1-t^{4}\right)\left(1-t^{6}\right)\left(1-t^{12}\right)\left(1-t^{12-2 N}\right)} \\
& D_{N, \delta}=t^{12 \delta} \frac{t^{25-2 N}+t^{11}+t^{9}+\cdots+t^{15-2 N}}{\left(1-t^{4}\right)\left(1-t^{6}\right)\left(1-t^{12}\right)\left(1-t^{12-2 N}\right)}
\end{aligned}
$$

Proof. Since $\operatorname{dim} M_{\nu}=0$ for $\nu<0$, we may make the computation slightly easier by summing over all $k \in \mathbb{Z}$ and using, for all $a \in \mathbb{Z}$, the identity $\sum_{k \in \mathbb{Z}} \operatorname{dim} M_{k-a} t^{k}=t^{a} /\left(\left(1-t^{4}\right)\left(1-t^{6}\right)\right)$.

$$
\begin{aligned}
E_{N, \delta} & =\sum_{k \text { even }} \sum_{j=0}^{\infty} \sum_{i=0}^{N j} \operatorname{dim} M_{k+2 i-12(j+\delta)} t^{k}, \\
& =\sum_{j=0}^{\infty} \sum_{i=0}^{N j}\left(\sum_{k \text { even }} \operatorname{dim} M_{k+2 i-12(j+\delta)} t^{k+2 i-12(j+\delta)}\right) t^{12(j+\delta)-2 i} \\
& =\frac{1}{\left(1-t^{4}\right)\left(1-t^{6}\right)} \sum_{j=0}^{\infty} \sum_{i=0}^{N j} t^{12(j+\delta)-2 i} \\
& =\frac{t^{12 \delta}}{\left(1-t^{4}\right)\left(1-t^{6}\right)} \sum_{i=0}^{\infty} \sum_{j=\ulcorner i / N\urcorner}^{\infty} t^{12 j-2 i} \\
& =\frac{t^{12 \delta}}{\left(1-t^{4}\right)\left(1-t^{6}\right)\left(1-t^{12}\right)} \sum_{i=0}^{\infty} \sum_{j=\ulcorner i / N\urcorner}^{\infty}\left(t^{12 j-2 i}-t^{12(j+1)-2 i}\right) \\
& =\frac{t^{12 \delta}}{\left(1-t^{4}\right)\left(1-t^{6}\right)\left(1-t^{12}\right)} \sum_{i=0}^{\infty} t^{12\ulcorner i / N\urcorner-2 i} .
\end{aligned}
$$


We finish by substituting $i=N \ell+\nu$ and evaluating

$$
\begin{aligned}
& \sum_{i=0}^{\infty} t^{12\ulcorner i / N\urcorner-2 i}=\sum_{\nu=0}^{N-1} \sum_{\ell=0}^{\infty} t^{12\left\ulcorner\frac{N \ell+\nu}{N}\right\urcorner-2(N \ell+\nu)}= \\
& \sum_{\ell=0}^{\infty} t^{12 \ell-2 N \ell}+\sum_{\nu=1}^{N-1} \sum_{\ell=0}^{\infty} t^{12(\ell+1)-2 N \ell-2 \nu}=\sum_{\ell=0}^{\infty} t^{(12-2 N) \ell}\left(1+\sum_{\nu=1}^{N-1} t^{12-2 \nu}\right) \\
& =\frac{1+t^{10}+t^{8}+\cdots+t^{14-2 N}}{1-t^{12-2 N}} .
\end{aligned}
$$

The proof for $D_{N, \delta}$ is quite similar.

$$
\begin{aligned}
D_{N, \delta} & =\sum_{k \text { odd }} \sum_{j=1}^{\infty} \sum_{i=1}^{N j-1} \operatorname{dim} M_{k-1+2 i-12(j+\delta)} t^{k}, \\
= & \sum_{j=1}^{\infty} \sum_{i=1}^{N j-1}\left(\sum_{k \text { odd }} \operatorname{dim} M_{k-1+2 i-12(j+\delta)} t^{k-1+2 i-12(j+\delta)}\right) t^{12(j+\delta)-2 i+1} \\
= & \frac{1}{\left(1-t^{4}\right)\left(1-t^{6}\right)} \sum_{j=1}^{\infty} \sum_{i=1}^{N j-1} t^{12(j+\delta)-2 i+1} \\
= & \frac{t^{12 \delta}}{\left(1-t^{4}\right)\left(1-t^{6}\right)} \sum_{i=1}^{\infty} \sum_{j=\ulcorner(i+1) / N\urcorner}^{\infty} t^{12 j-2 i+1} \\
= & \frac{t^{12 \delta}}{\left(1-t^{4}\right)\left(1-t^{6}\right)\left(1-t^{12}\right)} \sum_{i=1}^{\infty} \sum_{j=\ulcorner(i+1) / N\urcorner}^{\infty}\left(t^{12 j-2 i+1}-t^{12(j+1)-2 i+1}\right) \\
= & \frac{t^{12 \delta}}{\left(1-t^{4}\right)\left(1-t^{6}\right)\left(1-t^{12}\right)} \sum_{i=1}^{\infty} t^{12\ulcorner(i+1) / N\urcorner-2 i+1} .
\end{aligned}
$$

We finish by substituting $i=N \ell+\nu$ and evaluating

$$
\begin{aligned}
& \sum_{i=1}^{\infty} t^{12\ulcorner(i+1) / N\urcorner-2 i+1}=\sum_{\nu=1}^{N} \sum_{\ell=0}^{\infty} t^{12\left\ulcorner\frac{N \ell+\nu+1}{N}\right\urcorner-2(N \ell+\nu)+1}= \\
& \sum_{\nu=1}^{N-1} \sum_{\ell=0}^{\infty} t^{12(\ell+1)-2 N \ell-2 \nu+1}+\sum_{\ell=0}^{\infty} t^{12(\ell+2)-2(N \ell+N)+1} \\
= & \sum_{\ell=0}^{\infty} t^{(12-2 N) \ell}\left(\sum_{\nu=1}^{N-1} t^{13-2 \nu}+t^{25-2 N}\right) \\
= & \frac{t^{11}+t^{9}+\cdots+t^{15-2 N}+t^{25-2 N}}{1-t^{12-2 N}} .
\end{aligned}
$$


The proof for the case $N=1$ is similar and is given in Aoki [1].

Corollary 3.4. For $N \in\{2,3,4\}$ and $\epsilon \in\{-1,1\}$ or for $N=1$ and $\epsilon=1$, all the inequalities in the Estimate of Lemma 3.2 are equalities.

$\forall k \in \mathbb{Z}, \quad \mathrm{FJ}: M_{k}(K(N))^{\epsilon} \rightarrow \mathbb{M}_{k}(N)^{\epsilon}$ is an isomorphism.

$\forall k$ even, $\quad \operatorname{dim} J_{k, N j}(j+\delta)=\sum_{i=0}^{N j} \operatorname{dim} M_{k+2 i-12(j+\delta)}$,

$\forall k$ odd, $\quad \operatorname{dim} J_{k, N j}(j+\delta)=\sum_{i=1}^{N j-1} \operatorname{dim} M_{k-1+2 i-12(j+\delta)}$,

$$
\begin{aligned}
& \sum_{k=0}^{\infty} \operatorname{dim} M_{k}(K(N))^{+} t^{k}=E_{N, 0}+D_{N, 1}= \\
& \frac{1+t^{10}+t^{8}+\cdots+t^{14-2 N}+t^{12}\left(t^{11}+t^{9}+\cdots+t^{15-2 N}+t^{25-2 N}\right)}{\left(1-t^{4}\right)\left(1-t^{6}\right)\left(1-t^{12}\right)\left(1-t^{12-2 N}\right)} \\
& \sum_{k=0}^{\infty} \operatorname{dim} M_{k}(K(N))^{-} t^{k}=E_{N, 1}+D_{N, 0}= \\
& \frac{t^{12}\left(1+t^{10}+t^{8}+\cdots+t^{14-2 N}\right)+t^{11}+t^{9}+\cdots+t^{15-2 N}+t^{25-2 N}}{\left(1-t^{4}\right)\left(1-t^{6}\right)\left(1-t^{12}\right)\left(1-t^{12-2 N}\right)} .
\end{aligned}
$$

Proof. Rewriting the inequalities of Lemma 3.2 as $\operatorname{dim} M_{k}(K(N))^{+} \leq$ $\operatorname{coeff}\left(E_{N, 0}+D_{N, 1}, t^{k}\right)$ and as $\operatorname{dim} M_{k}(K(N))^{-} \leq \operatorname{coeff}\left(E_{N, 1}+D_{N, 0}, t^{k}\right)$, we have $\operatorname{dim} M_{k}(K(N))=\operatorname{dim} M_{k}(K(N))^{+}+\operatorname{dim} M_{k}(K(N))^{-} \leq$ $\operatorname{coeff}\left(E_{N, 0}+D_{N, 1}+E_{N, 1}+D_{N, 0}, t^{k}\right)$. If we can show equality here, we have $\operatorname{dim} M_{k}(K(N))^{+}=\operatorname{coeff}\left(E_{N, 0}+D_{N, 1}, t^{k}\right)$ and $\operatorname{dim} M_{k}(K(N))^{-}=$ $\operatorname{coeff}\left(E_{N, 1}+D_{N, 0}, t^{k}\right)$ and the proof is complete. However, the generating functions $\sum_{k \in \mathbb{Z}} \operatorname{dim} M_{k}(K(N)) t^{k}$ are known for $N=2,3$ and 4 and one checks equality with $E_{N, 0}+E_{N, 1}+D_{N, 0}+D_{N, 1}$.

\section{The Generating Function of $K(4)$}

For any natural number $t$, the paramodular group $K\left(t^{2}\right)$ is conjugate, by an element of $\operatorname{Sp}_{2}(\mathbb{Q})$, to the following group $\tilde{\Gamma}(t)$, which is a subgroup of $\Gamma_{2}$ containing the principal subgroup $\Gamma_{2}(t)$;

$$
\tilde{\Gamma}(t)=\left(\begin{array}{cccc}
* & t * & * & t * \\
t * & * & t * & * \\
* & t * & * & t * \\
t * & * & t * & *
\end{array}\right) \cap \operatorname{Sp}_{2}(\mathbb{Z}), \text { where } * \in \mathbb{Z} .
$$


Table $1 . \quad S_{6}$ cycles.

$\begin{array}{lllll}\frac{S_{3} \times S_{3}}{(1) \times(1)} & 1 & (1) & 1 & \frac{\left(1+t^{5}\right)\left(1-t^{8}\right)}{\left(1-t^{2}\right)^{5}} \\ (12) \times(1) & 3 & & & \\ (1) \times(12) & 3 & (12) & 6 & \frac{\left(1-t^{5}\right)\left(1-t^{8}\right)}{\left(1-t^{2}\right)^{2}\left(1+t^{2}\right)^{3}} \\ (12) \times(12) & 9 & (12)(34) & 9 & \frac{\left(1+t^{5}\right)\left(1-t^{8}\right)}{\left(1-t^{2}\right)^{3}\left(1+t^{2}\right)^{2}} \\ (123) \times(1) & 2 & & & \\ (1) \times(123) & 2 & (123) & 4 & \frac{\left(1+t^{5}\right)\left(1-t^{8}\right)}{\left(1-t^{2}\right)\left(1+t^{2}+t^{4}\right)^{2}} \\ (123) \times(12) & 6 & & & \frac{\left(1-t^{5}\right)\left(1-t^{8}\right)}{\left(1+t^{2}\right)\left(1-t^{2}+t^{4}\right)\left(1+t^{2}+t^{4}\right)} \\ (12) \times(123) & 6 & (12)(345) & 12 & \\ (123) \times(123) & 4 & (123)(456) & 4 & \frac{\left(1+t^{5}\right)\left(1-t^{8}\right)}{\left(1+t^{2}\right)^{3}\left(1+t^{2}+t^{4}\right)}\end{array}$

The proof is that $\operatorname{diag}\left(1, t, 1, t^{-1}\right) K\left(t^{2}\right) \operatorname{diag}\left(1, t^{-1}, 1, t\right)=\tilde{\Gamma}(t)$. In Igusa [14], we may find the generating function for the character $X_{k}$ of the representation of $\mathrm{Sp}_{2}\left(\mathbb{F}_{2}\right) \simeq \Gamma_{2} / \Gamma_{2}(2)$ acting on $M_{k}\left(\Gamma_{2}(2)\right)$. Since $\tilde{\Gamma}(2)$ contains the principal subgroup $\Gamma_{2}(2)$, Igusa's result allows us to calculate the generating function for $\tilde{\Gamma}(2)$ by the formula

$$
\sum_{k=0}^{\infty} \operatorname{dim} M_{k}(\tilde{\Gamma}(2)) t^{k}=\frac{1}{|G|} \sum_{M \in G} \sum_{k=0}^{\infty} X_{k}(M) t^{k},
$$

where $G=\tilde{\Gamma}(2) / \Gamma_{2}(2)$ is a finite group. Now $\mathrm{Sp}_{2}\left(\mathbb{F}_{2}\right)$ is isomorphic to the symmetric group $S_{6}$ via the permutation of the six odd theta characteristics and the group $G \simeq \mathrm{SL}_{2}\left(\mathbb{F}_{2}\right) \times \mathrm{SL}_{2}\left(\mathbb{F}_{2}\right)$ corresponds to a choice of $S_{3} \times S_{3} \subseteq S_{6}$ by the action of $\mathrm{SL}_{2}\left(\mathbb{F}_{2}\right)$ on the three even theta characteristics. We separate the elements $M \in G$ into conjugacy classes, which may be given by cycle types inside $S_{6}$, and give Igusa's computation (page 401, 14]) of $g(M ; t)=\sum_{k=0}^{\infty} X_{k}(M) t^{k}$ for these conjugacy classes. Table 1 lists the cycle types in both $S_{3} \times S_{3}$ and $S_{6}$ and gives the number of elements that have that cycle type. 
This gives $\sum_{k=0}^{\infty} \operatorname{dim} M_{k}(K(4)) t^{k}=\sum_{k=0}^{\infty} \operatorname{dim} M_{k}(\tilde{\Gamma}(2)) t^{k}=$

$$
\begin{aligned}
& \frac{1}{36}\{g((1) ; t)+6 g((12) ; t)+9 g((12)(34) ; t)+ \\
& 4 g((123) ; t)+12 g((12)(345) ; t)+4 g((123)(456) ; t)\} \\
& \quad=\frac{\left(1+t^{12}\right)\left(1+t^{6}+t^{7}+t^{8}+t^{9}+t^{10}+t^{11}+t^{17}\right)}{\left(1-t^{4}\right)^{2}\left(1-t^{6}\right)\left(1-t^{12}\right)} .
\end{aligned}
$$

We mention a good cross check now that we know $\operatorname{dim} M_{k}(K(4))$. We can show that $\operatorname{dim} J_{k, 4 j}^{\text {cusp }}(j)=\max \left\{\operatorname{dim} J_{k, 4 j}(j)-1,0\right\}$ by comparing the Taylor expansion and the theta expansion of Jacobi forms as in Eichler-Zagier [5]. By this, we can also give upper bounds for $\operatorname{dim} S_{k}(K(4))$. These upper bounds coincide with the true dimension of $S_{k}(K(4))$ computed from the known dimension of $M_{k}(K(4))$ and the dimension of the image of generalized $\Phi$-operator on the boundary.

$$
\text { 5. An Example: } \pi_{12} \circ \mathrm{FJ}: S^{4}(K(31)) \rightarrow \prod_{j=1}^{12} J_{4,31 j}^{\mathrm{CUSP}}
$$

Although the linear relations from the Involution $(\epsilon)$ condition are practical to implement on a computer, the growth condition is not. It is natural to wonder about the effect of omitting the growth condition and we work out one example with this in mind. In light of Theorem 2.2, when we compute formal power series over Jacobi forms satisfying the involution condition, either there will only be Fourier Jacobi expansions of paramodular forms or there will also be solutions with rapidly growing coefficients. We consider the subspaces $S_{4}(K(31))^{-}$ and

$$
\mathcal{S}=\left\{f \in S_{4}(K(31))^{+}: \operatorname{ord}_{\xi} \mathrm{FJ}(f) \geq 62\right\}
$$

for the following reasons: The dimensions $\operatorname{dim} J_{k, m}^{\text {cusp }}$ are known for $k \geq$ 2, see [5] 20, and so we only need to generate sufficiently many linearly independent elements of $J_{k, m}^{\text {cusp }}$ to compute inside this space. Especially in weight four, see [9], theta blocks are a convenient way to construct Jacobi forms. For $d \in \mathbb{N}^{8}$ with $d \cdot d=2 N$, we have $T(d)(\tau, z)=$ $\prod_{i=1}^{8} \vartheta\left(\tau, d_{i} z\right) \in J_{4, N}$; here $\vartheta(\tau, z)=\sum_{n \in \mathbb{Z}}(-1)^{n} q^{\frac{(2 n+1)^{2}}{8}} \zeta^{\frac{2 n+1}{2}}$. It is easy to see that $T(d)$ is a cusp form if $d$ has both even and odd entries. We select $K(p)$ for prime level $p$ because T. Ibukiyama [10] [12] has given $\operatorname{dim} S_{k}(K(p))$ for $k \geq 3$; this information allows us to measure our computations against a known dimension. For weight 4 , we have

$$
\begin{gathered}
\operatorname{dim} S_{4}(K(p))= \\
\frac{p^{2}}{576}+\frac{p}{8}-\frac{143}{576}+\left(\frac{p}{96}-\frac{1}{8}\right)\left(\frac{-1}{p}\right)+\frac{1}{8}\left(\frac{2}{p}\right)+\frac{1}{12}\left(\frac{3}{p}\right)+\frac{p}{36}\left(\frac{-3}{p}\right)
\end{gathered}
$$




$$
\operatorname{dim} J_{4, m}^{\text {cusp }}=\sum_{j=1}^{m}\left(\{\{4+2 j\}\}-\left\llcorner\left(j^{2} / 4 m\right)\right\lrcorner\right),
$$

where we let $\llcorner x\lrcorner=\max \{m \in \mathbb{Z}: m \leq x\}$ be the greatest integer function and where $\{\{k\}\}=\operatorname{dim} S_{k}\left(\mathrm{SL}_{2}(\mathbb{Z})\right)$.

V. Gritsenko has a lifting Grit $: J_{k, N}^{\text {cusp }} \rightarrow S_{k}(K(N))^{\epsilon}$ for $\epsilon=(-1)^{k}$ with the property that the Fourier Jacobi expansion of $\operatorname{Grit}(\phi)$ has leading term $\phi \xi^{N}$, see [7]. In selecting a generic example, we avoid these lifts because their Fourier coefficients satisfy special linear relations. The first prime $p$ for which the map Grit : $J_{4, p}^{\text {cusp }} \rightarrow S_{4}(K(p))$ does not surject is $p=31$; here Grit $\left(J_{4,31}^{\text {cusp }}\right)$ is five dimensional and $S_{4}(K(31))$ six. By subtracting off the Gritsenko lift of the leading Fourier Jacobi coefficient we have $S_{4}(K(31))^{+}=\operatorname{Grit}\left(J_{4,31}^{\text {cusp }}\right) \oplus \mathcal{S}$. We will compute 12 coefficients of the Fourier Jacobi expansions from $S_{4}(K(31))$ in accordance with the following Lemma, noting here that $\frac{k}{10} \frac{p^{2}+1}{p+1}=\frac{4}{10} \frac{31^{2}+1}{31+1}=12.025$.

Lemma 5.1. Let $p$ be a prime, $J, M, k \in \mathbb{N}$ and $\epsilon \in\{-1,1\}$. Let $\pi_{M}: \prod_{j=1}^{\infty} J_{k, p j} \rightarrow \prod_{j=1}^{\llcorner M / p\lrcorner} J_{k, p j}$ be projection. The map $\pi_{p J} \circ \mathrm{FJ}:$ $S_{k}(K(p))^{\epsilon} \rightarrow \prod_{j=1}^{J} J_{k, p j}^{\text {cusp }}$ injects for $J \geq\left\llcorner\frac{k}{10}\left(\frac{p^{2}+1}{p+1}\right)\right\lrcorner$.

Proof. For $T \in \mathcal{X}_{2}(p)=\left\{\left(\begin{array}{ll}a & b \\ b & c\end{array}\right)>0: a, 2 b, c \in \mathbb{Z}\right.$ and $\left.p \mid c\right\}$, define the Minimum function $m$ via $m(T)=\min _{x} T[x]$ over $x \in \mathbb{Z}^{2} \backslash\{0\}$. It is known that the $T \in \mathcal{X}_{2}(p)$ with $m(T) \leq \frac{k}{10} \frac{p^{2}+1}{p+1}$ are a determining set of Fourier coefficients for $S_{k}(K(p))^{\epsilon}$, see [16]. Consider $f \in S_{k}(K(p))^{\epsilon}$ such that

$$
\forall T=\left(\begin{array}{cc}
n & r / 2 \\
r / 2 & m
\end{array}\right) \in \mathcal{X}_{2}(p): \frac{m}{p} \leq \frac{k}{10} \frac{p^{2}+1}{p+1}, a(T ; f)=0 .
$$

We need to show that such $f$ vanish. Take any $T \in \mathcal{X}_{2}(p)$ satisfying $m(T) \leq \frac{k}{10} \frac{p^{2}+1}{p+1}$. By reduction we have $T=\left(\begin{array}{ll}a & b \\ b & c\end{array}\right)\left[\left(\begin{array}{ll}\alpha & \beta \\ \gamma & \delta\end{array}\right)\right]$ for some $\left(\begin{array}{ll}\alpha & \beta \\ \gamma & \delta\end{array}\right) \in \mathrm{GL}_{2}(\mathbb{Z})$ and $0 \leq 2 b \leq c \leq a$; in this case $c=m(T)$. If $p \mid \beta$ then $\left(\begin{array}{ll}\alpha & \beta \\ \gamma & \delta\end{array}\right) \in \hat{\Gamma}^{0}(p)$ and $a(T)= \pm a\left(\left(\begin{array}{ll}a & b \\ b & c\end{array}\right)\right)=0$ by (3) since $\frac{c}{p} \leq c \leq \frac{k}{10} \frac{p^{2}+1}{p+1}$. If $\beta$ is prime to $p$, let $r \in \mathbb{Z}$ solve $\beta r \equiv \delta \bmod p$; then $\sigma=\left(\begin{array}{ll}\alpha & \beta \\ \gamma & \delta\end{array}\right)^{-1}\left(\begin{array}{ll}0 & 1 \\ 1 & r\end{array}\right) \in \hat{\Gamma}^{0}(p)$ and we have $T[\sigma]=\left(\begin{array}{ll}a & b \\ b & c\end{array}\right)\left[\left(\begin{array}{ll}0 & 1 \\ 1 & r\end{array}\right)\right]=$ $\left(\begin{array}{cc}c & b+r c \\ b+r c & c r^{2}+2 b r+a\end{array}\right) \in \mathcal{X}_{2}(p)$ so that $p \mid\left(c r^{2}+2 b r+a\right)$. In this case

$$
a(T)=\operatorname{det}(\sigma)^{k} a(T[\sigma])=\epsilon \operatorname{det}(\sigma)^{k} a\left(\left(\begin{array}{cc}
\frac{c r^{2}+2 b r+a}{p} & -(c r+b) \\
-(c r+b) & p c
\end{array}\right)\right)=0
$$


by (3) because $\frac{p c}{p}=c \leq \frac{k}{10} \frac{p^{2}+1}{p+1}$. Since $a(T)=0$ for all $T$ with $m(T) \leq$ $\frac{k}{10} \frac{p^{2}+1}{p+1}$, we have $f=0$.

For $p=31$ and $k=4$, the following Proposition computes the first $J=12$ Jacobi form coefficients of any formal power series that satisfies the Involution $(\epsilon)$ condition and finds that they are all initial FourierJacobi expansions of paramodular cusp forms. This makes it at least plausible that the involution condition alone characterizes the Fourier Jacobi expansions from $S_{4}(K(31))^{\epsilon}$ from among all formal power series over Jacobi forms. And that is the point of this computation- to show that the growth condition may be superfluous.

Proposition 5.2. Let $k, p, J \in \mathbb{N}$ with $p$ prime. Define the subspaces

$$
\begin{aligned}
& A(J)=\left\{\Phi=\sum_{j=1}^{J} \phi_{j p} \xi^{j p} \in \prod_{j=1}^{J} J_{k, j p}^{\text {cusp }}: \Phi \text { satisfies Involution }(-)\right\} \text { and } \\
& B(J)=\left\{\Phi=\sum_{j=2}^{J} \phi_{j p} \xi^{j p} \in \prod_{j=1}^{J} J_{k, j p}^{\text {cusp }}: \Phi \text { satisfies Involution }(+)\right\} .
\end{aligned}
$$

For $k=4$ and $p=31$, the subspace $A(12)$ is trivial and the subspace $B(12)$ is one dimensional and is spanned by $\Phi_{0}=\psi_{62} \xi^{62}+\psi_{93} \xi^{93}+$ $\cdots+\psi_{12 \cdot 31} \xi^{12 \cdot 31}$ with initial expansions

$$
\begin{aligned}
& \psi_{62}=\mathbf{q}^{2}\left(-\zeta^{22}+7 \zeta^{21}-15 \zeta^{20}-3 \zeta^{19}+50 \zeta^{18}-37 \zeta^{17}-47 \zeta^{16}\right. \\
& +19 \zeta^{15}+74 \zeta^{14}+49 \zeta^{13}-163 \zeta^{12}-13 \zeta^{11}+67 \zeta^{10}+28 \zeta^{9}+108 \zeta^{8} \\
& -84 \zeta^{7}-106 \zeta^{6}-74 \zeta^{5}+114 \zeta^{4}+162 \zeta^{3}-84 \zeta^{2}-54 \zeta+6-54 / \zeta \\
& -84 / \zeta^{2}+162 / \zeta^{3}+114 / \zeta^{4}-74 / \zeta^{5}-106 / \zeta^{6}-84 / \zeta^{7}+108 / \zeta^{8} \\
& +28 / \zeta^{9}+67 / \zeta^{10}-13 / \zeta^{11}-163 / \zeta^{12}+49 / \zeta^{13}+74 / \zeta^{14}+19 / \zeta^{15} \\
& \left.-47 / \zeta^{16}-37 / \zeta^{17}+50 / \zeta^{18}-3 / \zeta^{19}-15 / \zeta^{20}+7 / \zeta^{21}-1 / \zeta^{22}\right) \\
& +\mathbf{q}^{3}\left(\zeta^{27}-5 \zeta^{26}+5 \zeta^{25}+11 \zeta^{24}-19 \zeta^{23}-2 \zeta^{22}-5 \zeta^{21}+21 \zeta^{20}+39 \zeta^{19}\right. \\
& -47 \zeta^{18}-5 \zeta^{17}-64 \zeta^{16}+19 \zeta^{15}+133 \zeta^{14}-25 \zeta^{13}+17 \zeta^{12}-131 \zeta^{11} \\
& -52 \zeta^{10}+71 \zeta^{9}-3 \zeta^{8}+159 \zeta^{7}-37 \zeta^{6}-49 \zeta^{5}-38 \zeta^{4}-86 \zeta^{3}+10 \zeta^{2} \\
& +26 \zeta+112+26 / \zeta+10 / \zeta^{2}-86 / \zeta^{3}-38 / \zeta^{4}-49 / \zeta^{5}-37 / \zeta^{6} \\
& +159 / \zeta^{7}-3 / \zeta^{8}+71 / \zeta^{9}-52 / \zeta^{10}-131 / \zeta^{11}+17 / \zeta^{12}-25 / \zeta^{13} \\
& +133 / \zeta^{14}+19 / \zeta^{15}-64 / \zeta^{16}-5 / \zeta^{17}-47 / \zeta^{18}+39 / \zeta^{19}+21 / \zeta^{20} \\
& \left.+5 / \zeta^{21}-2 / \zeta^{22}-19 / \zeta^{23}+11 / \zeta^{24}+5 / \zeta^{25}-5 / \zeta^{26}+1 / \zeta^{27}\right)+O\left(\mathbf{q}^{4}\right) ; \\
& \psi_{93}=\mathbf{q}^{2}\left(\operatorname{coeff}\left(\psi_{62}, q^{3}\right)\right)+O\left(\mathbf{q}^{3}\right) .
\end{aligned}
$$


Proof. It is convenient to denote $J_{k, m}^{\text {cusp }}(\nu)=\left\{\phi \in J_{k, m}^{\text {cusp }}:\right.$ ord $\left.\phi \geq \nu\right\}$. Let $\Phi=0 \cdot \xi^{31}+\phi_{62} \xi^{62}+\phi_{93} \xi^{93}+\cdots+\phi_{31 \nu} \xi^{31 \nu} \in B(\nu)$. The space $J_{4,62}^{\text {cusp }}$ is spanned by the 9 theta blocks $T(d)$ for $d=[1,1,1,1,2,4,6$, 8], [1, 1, 1, 2, 2, 2, 3, 10], [1, 1, 1, 2, 2, 4, 4, 9], [1, 1, 1, 2, 3, 6, 6, 6], $[1,1,2,2,2,2,5,9],[1,1,2,4,4,5,5,6],[1,2,2,2,2,3,7,7],[1,3$, $4,4,4,4,5,5],[2,2,2,2,3,3,3,9]$. The Involution(+) condition tells us that for all $\left(\begin{array}{cc}n & r / 2 \\ r / 2 & m\end{array}\right) \in \mathcal{X}_{2}(31)$ we have $c\left(n, r ; \phi_{m}\right)=c\left(\frac{m}{31},-r ; \phi_{31 n}\right)$. Setting $n=1$ and $m=62$ in condition Involution( + ), we have

$$
c\left(1, r ; \phi_{62}\right)=c\left(2,-r ; \phi_{31}\right)=c(2,-r ; 0)=0,
$$

so that the $q^{1}$-coefficients of $\phi_{62}$ vanish. The subspace $J_{4,62}^{\text {cusp }}(2)$ is spanned by one element, $\psi_{62}$, which is the following linear combination of the above nine theta blocks: $\psi_{62}=(-3,-5,-1,-2,-1,0,1,0,1)$. $\left(T\left(d_{1}\right), \ldots, T\left(d_{9}\right)\right)$. The initial expansion of $\psi_{62}$ is as given above. Thus $\phi_{62}$ is some multiple of $\psi_{62}$, say $\phi_{62}=\alpha \psi_{62}$ for $\alpha \in \mathbb{C}$, and the subspace $B(2)$ is at most one dimensional.

The space $J_{4,93}^{\text {cusp }}$ is spanned by the 16 theta blocks $T(c)$ for $c=[1$, $1,1,1,1,1,6,12],[1,1,1,1,1,6,8,9],[1,1,1,1,2,3,5,12]$, [1, $1,1,1,2,4,9,9],[1,1,1,1,4,6,7,9],[1,1,1,3,5,6,7,8],[1,1$, $2,2,2,6,6,10],[1,1,2,3,3,3,3,12],[1,1,2,3,3,4,5,11],[1,1$, $2,6,6,6,6,6],[1,2,3,3,3,3,8,9],[1,3,4,4,6,6,6,6],[2,2,2$, $2,2,2,9,9],[2,2,2,2,2,3,6,11],[2,3,3,3,3,3,4,11],[3,4,5$, $5,5,5,5,6]$. For $n=1$ and $m=93$ the Involution( + ) conditions are $c\left(1, r ; \phi_{93}\right)=c\left(3,-r ; \phi_{31}\right)=0$ so that $\phi_{93} \in J_{4,93}^{\text {cusp }}(2)$. The subspace $J_{4,93}^{\text {cusp }}(3)$ is trivial and the subspace $J_{4,93}^{\text {cusp }}(2)$ is spanned by the following four linear combinations of theta blocks:

$$
\begin{aligned}
& Q_{1}=(-1,-1,-6,-6,-4,-1,0,-1,2,0,1,0,0,0,0,0) \cdot\left(T\left(c_{1}\right), \ldots, T\left(c_{16}\right)\right), \\
& Q_{2}=(-2,-1,-9,-6,-3,0,-1,-1,3,0,0,0,1,0,0,0) \cdot\left(T\left(c_{1}\right), \ldots, T\left(c_{16}\right)\right), \\
& Q_{3}=(-1,-1,-4,-2,0,0,1,1,-2,0,0,0,0,1,0,0) \cdot\left(T\left(c_{1}\right), \ldots, T\left(c_{16}\right)\right), \\
& Q_{4}=(1,0,1,3,2,-1,0,-1,-5,0,0,0,0,0,1,0) \cdot\left(T\left(c_{1}\right), \ldots, T\left(c_{16}\right)\right) .
\end{aligned}
$$

Some Fourier coefficients for these $Q_{i}$ are in Table 2. We use the Involution $(+)$ condition for $n=2$ and $m=93$ to find the $q^{2}$-coefficients of $\phi_{93}$.

$$
c\left(2, r ; \phi_{93}\right)=c\left(3,-r ; \phi_{62}\right)=\alpha c\left(3,-r ; \psi_{62}\right)
$$

The coefficients $c\left(3,-r ; \psi_{62}\right)$ are known and displayed in the statement of the Proposition. The unique element $\phi_{93} \in J_{4,93}^{\text {cusp }}(2)$ satisfying equation (4) is $\alpha \psi_{93}$ where $\psi_{93}=-Q_{1}-Q_{4}$. This shows that the subspace 
$B(3)$ is at most one dimensional. Continuing in this way on a computer, we showed that $J_{4,31 j}^{\text {cusp }}(j)=\{0\}$ for $j=3, \ldots, 12$ and hence that $\operatorname{dim} B(12) \leq 1$.

We discuss the minus space. The space $J_{4,31}^{\text {cusp }}$ is spanned by 5 theta blocks $T(b)$ for $b=[1,1,1,1,1,2,2,7],[1,1,1,1,1,4,4,5]$, $[1,1,1,1,2,2,5,5],[1,1,2,2,2,4,4,4],[2,2,3,3,3,3,3,3]$. For even weights, the Involution $(-)$ conditions are quite restrictive. We have $c\left(j, r ; \phi_{31 j}\right)=-c\left(j,-r ; \phi_{31 j}\right)$, so that the $q^{j}$-coefficients of $\phi_{31 j}$ must vanish. However, the $q^{1}$-coefficients of the five theta blocks $T\left(b_{i}\right)$ are already linearly independent, so $A(1)$ is trivial. Now that we know that the first Jacobi coefficient vanishes, by the same reasoning as for the plus space, the only possible element of $A(2)$ is a multiple of $\psi_{62}$; however the extra condition that the $q^{2}$-coefficients of $\phi_{62}$ vanish shows that $A(2)$ is trivial. The triviality of $A(12)$ now follows from $J_{4,31 j}^{\text {cusp }}(j)=\{0\}$ for $j=3, \ldots, 12$.

By Theorem 2.2 we have a map $\pi_{12.31} \circ \mathrm{FJ}: S_{4}(K(31))^{-} \rightarrow A(12)$ and, by Lemma 5.1, this map is injective; hence $S_{4}(K(31))^{-}$is trivial. From Ibukiyama's result, $\operatorname{dim} S_{4}(K(31))=6$, we may conclude that $\operatorname{dim} S_{4}(K(31))^{+}=6$ and $\operatorname{dim} \mathcal{S}=1$. Therefore $\Phi_{0} \in \pi_{12.31} \mathrm{FJ}(\mathcal{S}) \subseteq$ $B(12)$ and $\operatorname{dim} B(12)=1$.

From another point of view, the merit of the preceding computations consists in providing upper bounds for the dimension of spaces of paramodular cusp forms. In this particular case, relying on Ibukiyama's dimension formula for the existence of forms, we have shown the following Corollary.

Corollary 5.3. $\operatorname{dim} S_{4}(K(31))^{+}=6$ and $\operatorname{dim} S_{4}(K(31))^{-}=0$.

\section{FinAL REMARKS}

We conclude by comparing the Involution condition with the following weaker inequality; for general $N$, we cannot even show that the right hand side is finite:

$$
\operatorname{dim} S_{k}(K(N))^{(-1)^{k}} \leq \sum_{j=1}^{\infty} \operatorname{dim} J_{k, N j}^{\text {cusp }}(j) .
$$


Table 3

Values of $\operatorname{dim} J_{k, 5 j}^{\text {cusp }}(j)$

\begin{tabular}{|c|c|c|c|c|c|c|}
\hline$k \backslash^{j}$ & 1 & 2 & 3 & 4 & $\sum_{j=1}^{\infty} \operatorname{dim} J_{k, 5 j}^{\text {cusp }}(j)$ & $\operatorname{dim} S_{k}(K(5))^{(-1)^{k}}$ \\
\hline 1 & & & & & 0 & 0 \\
\hline 2 & & & & & 0 & 0 \\
\hline 3 & & & & & 0 & 0 \\
\hline 4 & & & & & 0 & 0 \\
\hline 5 & 1 & & & & 1 & 1 \\
\hline 6 & 1 & & & & 1 & 1 \\
\hline 7 & 1 & & & & 1 & 1 \\
\hline 8 & 2 & 0 & & & 2 & 2 \\
\hline 9 & 2 & 0 & & & 2 & 2 \\
\hline 10 & 3 & 1 & 0 & & 4 & 4 \\
\hline 11 & 3 & 1 & 0 & & 4 & 4 \\
\hline 12 & 4 & 2 & 1 & & 7 & 6 \\
\hline 13 & 3 & 2 & 1 & 0 & 6 & 5 \\
\hline 14 & 5 & 3 & 2 & 0 & 10 & 8 \\
\hline 15 & 4 & 3 & 2 & 0 & 9 & 8 \\
\hline
\end{tabular}

For the case $N=31$ and $k=4$ we have demonstrated the equality $\operatorname{dim} S_{4}(K(31))^{+}=\sum_{j=1}^{20} \operatorname{dim} J_{4,31 j}^{\text {cusp }}(j)$ or $6=5+1+0+0+\cdots+0$. However tempting it may be to replace the 20 by $\infty$, we cannot be sure about that equality because we have only computed $\operatorname{dim} J_{4,31 j}^{\text {cusp }}(j)=0$ for $3 \leq j \leq 20$. We can, however, be certain about inequalities; for example with $N=29$ and $k=4$, we can show the inequality $\operatorname{dim} S_{4}(K(29))^{+}<\sum_{j=1}^{\infty} \operatorname{dim} J_{4,29 j}^{\text {cusp }}(j)$ or $5<5+1+0+\ldots$. The space $J_{4,58}^{\text {cusp }}(2)$ is one dimensional, spanned by $\Psi$ say, but there is no element in $J_{4,87}^{\text {cusp }}(2)$ whose $q^{2}$-terms equal the $q^{3}$-terms of $\Psi$. Hence there does exists a $\Phi=\Psi \xi^{2}$ satisfying the Involution(+) conditions to second order that is not the initial Fourier Jacobi expansion of any paramodular cusp form from $S_{4}(K(29))^{+}$. All the $\Phi$ that satisfy the Involution(+) 
condition to third order, however, are the initial Fourier Jacobi expansions of paramodular cusp forms from $S_{4}(K(29))^{+}$. Hence, again in this example, the Involution condition continues to compute the space $S_{4}(K(29))^{+}$correctly even when the inequality (5) is strict.

One case where the convergence of the series $\sum_{j=1}^{\infty} \operatorname{dim} J_{k, N j}^{\text {cusp }}(j)$ is known for all weights $k$ is $N=5$. Here the sum need only be taken to $j=\llcorner k / 2\lrcorner$ because ord $\phi \leq(k+2 m) / 12$ for $\phi \in J_{k, m}$. A more refined estimate of Gritsenko and Hulek [9] shows that $j \leq\llcorner(3 k-6) / 8\lrcorner$ suffices when $N=5$. Since $N=5$ is also the first level where the inequality (5) can be strict, it is of some interest to ponder this data. Table 3 gives the values of $\operatorname{dim} J_{k, 5 j}^{\text {cusp }}(j)$ for $1 \leq k \leq 15$ and for $j \leq\llcorner(3 k-6) / 8\lrcorner$. These were computed by using theta blocks to span the spaces of Jacobi forms. For weights $k \leq 15$, the dimensions of $S_{k}(K(5))^{ \pm}$in Table 3 may be found in a manner similar to that used to prove Corollary 5.3. One sees in Table 3 that the inequality (5) is already strict for weight $k=12$ and hence that the method that was used for $1 \leq N \leq 4$ to prove the surjectivity of FJ : $M_{k}(K(N))^{\epsilon} \rightarrow \mathbb{M}_{k}(N)^{\epsilon}$ for all weights will not work for $N=5$. 
Table 2

\begin{tabular}{|ccccc|}
\hline$r$ & $c\left(2, r ; Q_{1}\right)$ & $c\left(2, r ; Q_{2}\right)$ & $c\left(2, r ; Q_{3}\right)$ & $c\left(2, r ; Q_{4}\right)$ \\
\hline 0 & 114 & 300 & 6 & -226 \\
1 & -38 & -145 & -69 & 12 \\
2 & 14 & -47 & 89 & -24 \\
3 & -60 & -1 & 41 & 146 \\
4 & -34 & 84 & -72 & 72 \\
\hline 5 & 40 & -69 & -53 & 9 \\
6 & 65 & -27 & 41 & -28 \\
7 & 15 & 209 & 74 & -174 \\
8 & -42 & -113 & 0 & 45 \\
9 & -49 & -137 & -103 & -22 \\
\hline 10 & -65 & -72 & -49 & 117 \\
11 & 137 & 303 & 190 & -6 \\
12 & -33 & -93 & -55 & 16 \\
13 & 61 & -44 & -42 & -36 \\
14 & -79 & -10 & -72 & -54 \\
\hline
\end{tabular}

\section{REFERENCES}

[1] H. Aoki: Estimating Siegel modular forms of genus 2 using Jacobi forms, J. Math. Kyoto Univ. (JMKYAZ) 40-3 (2000), 581-588.

[2] H. Aoki: Estimating the dimension of the space of Siegel modular forms of genus 2 with level 2 and 3, Habuka, (2001), 1-6.

[3] O. Delzeith: Paramodulmannigfaltigkeiten von allgemeinem Typ, Inaugural Dissertation der Ruprecht-Karls-Universitaet Heidelberg, 1995.

[4] T. Dern: Paramodular forms of degree 2 and level 3, Comment. Math. Univ, St. Paul. 51, No. 2 (2002), 157-194.

[5] M. Eichler and D. Zagier: The theory of Jacobi forms, Progress in Mathematics, Vol. 55, Birkhäuser Verlag, 1985.

[6] Freitag, E.: Siegelsche Modulfunktionen, Grundlehren der mathematischen Wissenschaften, Band 254, Berlin-Heidelberg-New York: Springer-Verlag, 1983.

[7] V. Gritsenko: Arithmetical lifting and its applications, Number Theory, Paris (1992-93), 103-126 
[8] V. Gritsenko: Irrationality of the moduli spaces of polarized abelian surfaces, The International Mathematics Research Notices 6, (1994), 235-243.

[9] V. Gritsenko, K. Hulek: Commutator Coverings of Siegel Threefolds, Duke Math. Jour. 94 (1998), 509-542.

[10] T. Ibukiyama: On relations of dimensions of automorphic forms of $\operatorname{Sp}(2, \mathbf{R})$ and its compact twist Sp(2) (I). Advanced Studies in Pure Math. 7(1985), 7-29.

[11] T. Ibukiyama: On some alternating sums of dimemsions of Siegel modular forms of general degree and cusp configurations. J. Fac. Sci. Univ. Tokyo Sect. IA Math. 40(1993), no.2, 245-283.

[12] T. Ibukiyama : Dimension formulas of Siegel modular forms of weight 3 and supersingular abelian surfaces, Siegel Modular Forms and Abelian Varieties, Proceedings of the 4-th Spring Conference on Modular Forms and Related Topics (2007), 39-60.

[13] T. Ibukiyama, F. Onodera: On the Graded Ring of Modular Forms of the Siegel Paramodular Group of Level 2, Abh. Math. Sem. hamburg 67 (1997), 297-305.

[14] J. Igusa: On Siegel Modular Forms of Genus Two (II), Amer. J. Math. 86 (no. 2) (1964), 392-412.

[15] Poor, C., Yuen, D.: Computations of spaces of Siegel modular cusp forms, J. Math. Soc. Japan 59, No. 1 (2007), 185-222.

[16] Poor, C., Yuen, D.: Paramodular cusp forms, preprint (2009), 1-61. arXiv:0912.0049v1 [math.NT]

[17] Poor, C., Yuen, D.: The Cusp Structure of the Paramodular Groups for Degree Two, preprint (2011), 1-27.

[18] Reefschläger, H.: Berechnung Der Anzahl Der 1-Spitzen Der Paramodularen Gruppen 2-ten Grades, Dissertation der Universität Göttingen, 1973.

[19] Satake, I.: Surjectivité globale de l'opérateur $\Phi$, Fonctions Automorphes, Séminaire H. Cartan, E. N. S. 2 Exposé 16, 1-17, (1957/58). Secrétariat mathématique, 11 rue Pierre Curie, Paris 5e.

[20] N. Skoruppa, E. Zagier: Jacobi forms and a certain space of modular forms, Invent. Math. 94 (1988), 113-146. 
Table 2 (continued)

\begin{tabular}{|ccccc|}
\hline$r$ & $c\left(2, r ; Q_{1}\right)$ & $c\left(2, r ; Q_{2}\right)$ & $c\left(2, r ; Q_{3}\right)$ & $c\left(2, r ; Q_{4}\right)$ \\
\hline 15 & -42 & 0 & 67 & 23 \\
16 & 67 & 30 & 101 & -3 \\
17 & -40 & -99 & -122 & 45 \\
18 & 73 & 149 & 19 & -26 \\
19 & -57 & -55 & -3 & 18 \\
\hline 20 & 3 & -23 & 31 & -24 \\
21 & 7 & -6 & -5 & -2 \\
22 & -7 & 9 & -28 & 9 \\
23 & 19 & 30 & 24 & 0 \\
24 & -18 & -35 & -8 & 7 \\
\hline 25 & 7 & 14 & 1 & -12 \\
26 & -1 & -2 & 0 & 6 \\
27 & 0 & 0 & 0 & -1 \\
\hline
\end{tabular}

Department of Mathematics, Graduate School of Mathematics, OsAKa University, MachiKaneyama 1-1, TOYONAKA, Osaka, 560-0043 JAPAN

E-mail address: ibukiyam@math.sci.osaka-u.ac.jp

Department of Mathematics, Fordham University, Bronx, Ny 10458

E-mail address: poor@fordham.edu

Department of Mathematics and Computer Science, Lake Forest College, 555 N. Sheridan Rd., Lake Forest, IL 60045

E-mail address: yuen@lakeforest.edu 